\title{
Persistent Persister Misperceptions
}

\author{
Jun-Seob Kim and Thomas K. Wood* \\ Department of Chemical Engineering, Pennsylvania State University, University Park, PA, USA
}

Persister cells survive antibiotic treatment due to their lack of metabolism, rather than through genetic change, as shown via four seminal experiments conducted by the discoverers of the phenotype (Hobby et al., 1942; Bigger, 1944). Unfortunately, over seven decades of persister cell research, the literature has been populated by misperceptions that do not withstand scrutiny. This opinion piece examines some of those misunderstandings in the literature with the hope that by shining some light on these inaccuracies, the field may be advanced and subsequent manuscripts may be reviewed more critically.

Keywords: persister cells, antimicrobial tolerance

\section{REVEIW}

Persister cells survive antibiotic treatments by reducing their metabolism; i.e., rather than by mounting an active response to the antibiotic stress, such as activating efflux pumps and rather than undergoing mutation, they survive antibiotics by becoming dormant. By dormant, the experimental evidence indicates persister cells are non-growing (Hobby et al., 1942; Bigger, 1944) and lack transcription, translation, and proton-motive force (Kwan et al., 2013). Furthermore, given the widespread use of the synonym "tolerant" and its contrived distinction with persistence (Brauner et al., 2016), perhaps a working definition is best: persister cells are those cells that remain after the actively respiring cells are vanquished by antibiotics (usually seen as an unchanging or slowly decreasing concentration of viable cells). In the following paragraphs, each misperception about persistence is shown in bold followed by a discussion which examines it.

Ghent University, Belgiu

Bhaskar Chandra Mohan Ramisetty,

SASTRA University, India

*Correspondence:

Thomas K. Wood twood@engr.psu.edu

Specialty section: This article was submitted to Antimicrobials, Resistance and Chemotherapy,

a section of the journal

Frontiers in Microbiology

Received: 25 November 2016

Accepted: 19 December 2016

Published: 27 December 2016

\section{Misperception 1: Persister Cells Are Not Dormant}

When Hobby et al. (1942) first described persister cells, they showed that penicillin was ineffective against non-growing (cold) Staphylococcus aureus cells; i.e., persister cells are dormant. Bigger (Bigger, 1944) then confirmed that persister cells are dormant by showing (i) lowering the culture temperature to stop growth generates more persisters to penicillin, (ii) stopping growth by removing nutrients generates more persisters, and (iii) ceasing growth by adding boric acid at bacteriostatic concentrations generates more persisters.

Later research corroborated that persister cells are metabolically inactive; for example, Shah et al. (2006) found that metabolically inactive cells were more tolerant to the fluoroquinolone ofloxacin (although the sorting process itself in non-nutritive medium may have introduced artifacts), and Kwan et al. (2013) found that cells lacking protein synthesis, via pre-treatment with rifampin to stop transcription, with tetracycline to stop translation or with carbonyl cyanide $m$-chlorophenylhydrazone to halt ATP production, become persister cells. These three pre-treatments convert an initial population of $0.01 \%$ persisters to up to approximately $80 \%$ persisters (a 10,000-fold increase in persister cells). Hence, persister cells are predominantly dormant, although there may be a gradient in their metabolic activity, as they clearly must reduce their metabolism. 
In contrast, Orman and Brynildsen (2013) misinterpreted a set of fluorescence-activated cell sorting (FACS) experiments to claim E. coli persister cells were not necessarily dormant when they likely inadvertently carried over high numbers of dormant persister cells into their FACS experiments (Wood et al., 2013). Furthermore, the statistical errors in their technique were greater than the number of cells they used to make their conclusions, and the bulk of their data indicated persister cells are inactive. Paradoxically, the same group now claims, using more complicated FACS approaches, that persister cells are metabolically inactive as they write, "we used Persister-FACSeq on a library of reporters to study gene expression distributions in non-growing Escherichia coli, and found that persistence to ofloxacin is inversely correlated with the capacity of nongrowing cells to synthesize protein" (Henry and Brynildsen, 2016).

Other groups continue to claim persister cells are metabolically active, but there is usually a flaw in their procedures that nullifies their conclusion. For example, one common mistake is to convert persister cells into actively growing cells by providing a carbon source. This mistake was made by Orman and Brynildsen (2013) since the authors collected their E. coli persister cells by FACS but then mixed them into rich medium, which likely activated the formerly persister cells. Similarly, this mistake led to the incorrect conclusion that glycolysis intermediates "potentiate" the killing of persister cells by aminoglycoside antibiotics when in fact the authors merely woke the persister cells with nutrients and were studying actively growing cells, not persister cells, as evidenced by their supplemental data which showed the cells they were claiming to be persisters were only growing (Allison et al., 2011).

Pu et al. (2016) concluded that persister cells utilize the TolC efflux pump, may also have been studying actively growing cells since it is well-known that active cells utilize efflux pumps in an effort to survive antibiotic treatments. This speculation is based on the fact that the authors indicated in their supplemental material that they treated with antibiotics then washed with "M9 minimal medium" which presumably has glucose and then incubated with the fluorescent antibiotic BOCILLIN FL, so persister cells would have been converted to actively growing cells by the M9 minimal medium due to the presence of a carbon source. Furthermore, their conclusion that persister cells have greater efflux would be incorrect if the persister cells have less penicillin binding proteins (PBPs) due to their reduced protein production; i.e., the reduced fluorescence that was used to indicate more active efflux may be due to an artifact of lower PBPs in the persister cells since BOCILLIN FL binds PBPs.

For the work of Wakamoto et al. (2013), there is also an artifact. In this work, cells that were not killed by the prodrug isoniazid were indeed metabolically active. However, since isoniazid must be activated by catalase KatG, the metabolically active cells tolerant of isoniazid simply had low levels of KatG. Hence; the metabolic activity observed for cells surviving isoniazid cannot be concluded to be related to metabolic activity in persister cells (Wood et al., 2013).

\section{Misperception 2: Guanosine Tetraphosphate (ppGpp) Activates Lon Protease to Increase Persistence}

In regard to a mechanism of persister cell formation, it has been proposed that the ppGpp that mediates the stringent response activates Lon protease through polyphosphate, then Lon inactivates antitoxins of toxin/antitoxin (TA) systems, freeing the toxin to reduce metabolism (Maisonneuve et al., 2013). Since ppGpp was first linked to persistence in 2003 via the HipA toxin (Korch et al., 2003), the only novel part of this model is the connection of ppGpp to Lon protease via polyphosphate. However, one of the many flaws in this model is that at least in vitro, both ppGpp and polyphosphate deactivate Lon protease, rather than activate it (Osbourne et al., 2014). Furthermore, lon deletions in other labs have had no effect on persistence with aminoglycosides or $\beta$-lactams (Shan et al., 2015; Chowdhury et al., 2016a). Similarly, if the enzyme that produces polyphosphate, $\mathrm{PpK}$, is inactivated, the number of persisters are not altered significantly (Shan et al., 2015). Critically, in cells that no longer produce ppGpp, persister cells are still generated (Chowdhury et al., 2016a) so there are clearly other methods by which the cell becomes persistent. Also, this model does not hold for many type II TA systems since the authors subsequently published that it was not valid for toxins RelE, MazF, and YafO (Germain et al., 2015) and others showed it is not valid for the YoeB/YefM TA system (Ramisetty et al., 2016). Therefore, this flawed model appears to hold for the HipA toxin but a highly flawed system (Germain et al., 2015) was used to show its relevance for this TA system: both the flawed strain in which 10 type II TA systems were deleted ("delta 10"; see Misperception 3 below) and a non-toxic toxin was used (see Misperception 5 below). Additionally, this model is not relevant for type I (Verstraeten et al., 2015) or type III TA systems.

\section{Misperception 3: It Is Necessary to Delete Multiple TA Systems to See an Effect on Persistence}

This misperception is frequently propagated, even by authors who have proved it false. Critically, by deleting a single TA locus, several authors have shown that persistence is reduced; for example, a reduction in persistence upon deleting a single TA locus has been shown for $m q s R$ and $m q s R A$ (Kim and Wood, 2010; Luidalepp et al., 2011), tisAB/istR (Dörr et al., 2010), and yafQ (Harrison et al., 2009). Therefore, it is not necessary to reduce several TA loci simultaneously to see an impact on persistence as has been claimed (Maisonneuve et al., 2011). Inexplicably, the laboratory that claimed (incorrectly) to report the first single deletion that reduces persistence (Dörr et al., 2010), who wrote "Here, we show that a knockout of a particular SOS-TA locus, tisAB/istR, had a sharply decreased level of persisters tolerant to ciprofloxacin," now claim (Conlon et al., 2016) that multiple TA systems must be made to reduce persistence: "Although deletion of individual interferases has no phenotype, a knockout of ten TAs produced a decrease in persisters in both a growing culture and in the stationary phase." 
Since toxins of TA systems have different targets, indubitably, deletions of different TA systems should affect persistence to a different degree. Hence, it may be possible to delete some TA systems and see no effect on persistence but it is incorrect to indicate that it is necessary to delete more than one TA system to see an effect on persistence. Part of this misperception may be related to a failure to recognize that TA systems have other roles in different strains, such a phage inhibition (Pecota and Wood, 1996). Hence, not all TA systems are necessarily related to persistence.

\section{Misperception 4: The E. coli Delta 10 Strain Is a Good Tool for Studying Persister Cells}

At a time when it was already known that E. coli contains many more than 10 TA systems, a report was made about the impact of these 10 TA systems on persistence as well as the role of Lon protease in persistence (Maisonneuve et al., 2011); in this manuscript, the authors deleted 10 TA systems to create a "delta 10 " strain. Since E. coli has at least 39 TA systems (Tan et al., 2011; Yamaguchi et al., 2011; Wang et al., 2012; Guo et al., 2014), if only 10 of these are deleted, one clearly cannot claim much with confidence in regard to the effect of TA systems on cell physiology since at least 29 other TA systems that may potentially affect the cell. Furthermore, it was announced by the group that constructed that delta 10 strain at the 2015 American Society for Microbiology Meeting in New Orleans that the delta 10 strain contains mutations beyond those related to the TA systems that were deleted; these problems in its construction also invalidate its use. So use of the delta 10 strain suffers from two major flaws: there are at least 29 other functional TA systems in E. coli and the delta 10 strain has mutations beyond the deletion of the 10 TA systems that make a comparison to the wild-type invalid.

\section{Misperception 5: The Non-toxic HipA7 Variant Is a Suitable Tool for Persister Research}

Although the link between TA systems and persister cells was first established 33 years ago (in 1983) through the HipA/HipB TA system through the gain of function hipA7 mutation that increases persistence (Moyed and Bertrand, 1983), there are problems with this system. Notably, the HipA7 variant (containing the amino acid substitutions G22S and D291A) is not toxic (Korch et al., 2003). Therefore, the many persister-related experiments using this variant are not studying an active toxin and conclusions from these studies may be related to persistence but they are not necessarily related to TA systems. For example, hipA7 mutations have been found in antibiotic-tolerant E. coli strains that cause urinary tract infections; yet, activity of the HipA toxin variant after the G22S substitution was not shown (Schumacher et al., 2015). One group has even claimed recently that "Thus, slow growth per se does not induce persistence in the absence of TA-encoded toxins"; yet, their methods include the use of both this non-toxic allele (hipA7) and the flawed delta 10 host (Germain et al., 2015), so their results are unreliable. Therefore, given the artifacts associated with the hipA7 strain, the persister field would be better served by using other models in which an active toxin of a TA system is utilized.

\section{Misperception 6: Persister Cells Are Generated Primarily Spontaneously}

The single cell experiments showing stochastic or spontaneous generation of persister cells were based on the hipA7 strain (Balaban et al., 2004) that does not produce an active toxin (see Misperception 5 above) so the relevance of stochasticity and persistence is questionable. This paper also showed that persister cells were continuously generated in the exponential phase using this suspect strain (Balaban et al., 2004), a result that has been refuted by several groups who showed persister cells are absent from exponentially growing cells, if one is careful not to carry over persisters in the inoculum (Keren et al., 2004; Orman and Brynildsen, 2013). It also does not make sense physiologically to make persister cells spontaneously during times of an abundance of nutrients.

Kwan et al. (2013) demonstrated that pre-treatment with the antibiotics such as rifampicin and tetracycline converts nearly all the $E$. coli cells to the persister state; hence, persister cells go from a small subpopulation to being the main phenotype. These results were corroborated by Van den Bergh et al. (2016) who also demonstrated that pretreatment with antibiotics converts cells into the persister state. Others have also shown that environmental factors influence the persister state by using the antibiotic ciprofloxacin (Dörr et al., 2010), the interkingdom signal indole (Vega et al., 2012; Hu et al., 2015; Kwan et al., 2015b), and Pseudomonas aeruginosa quorum sensing molecules (Möker et al., 2010). Hence, although persister cells may arise spontaneously in small numbers (Balaban et al., 2004), the more germane physiological origin is probably related to the response of cells to stress.

\section{Misperception 7: Few Methods Exist to Kill Persister Cells}

This idea is false as there are many methods now that are effective against persister cells (Wood, 2016). Even in 2016, it has been stated that "Currently, few methods exist to kill persisters, and none are yet clinically developed" (Henry and Brynildsen, 2016). This statement is at best misleading since; for example, mitomycin C (Kwan et al., 2015a; Cruz-Muñiz et al., 2016) and cisplatin (Chowdhury et al., 2016b) have been found to kill persister cells very effectively (by crosslinking DNA while cells sleep). Although both compounds have been approved for use clinically for cancer treatments, they have not been approved for treating bacterial infections.

\section{Misperception 8: cAMP Increases Persistence}

Since cell populations change by orders of magnitude with antimicrobials, many erroneous results have been published based on relatively small changes in persister cell populations. For example, the Brynildsen group reported that exogenous (8 mM) cAMP increases persistence by 20 -fold (a relatively 
modest change in the persister field; Amato et al., 2013). In contrast, others have found that reducing internal cAMP concentrations via three complimentary methods has the opposite effect: (i) degrading cAMP with the cAMP-specific phosphodiesterase CpdA increases persistence 235-fold and an immunoassay confirmed cAMP levels were reduced by 323 -fold, (ii) inactivating the single adenylate cyclase that forms cAMP via the cyaA mutation increases persistence 19-fold, and (iii) degrading cAMP via non-specific phosphatase DosP increases persistence 4200-fold (Kwan et al., 2015b). Hence, for relatively small changes in persistence (20-fold or less), multiple lines of evidence are required before conclusions may be made with confidence.

\section{Misperception 9: It Is Difficult to Isolate Persister Cells as an Abundant Phenotype}

It has been argued that there is "an inability to isolate persisters from other more abundant cell types" (Henry and Brynildsen, 2016). This statement is highly inaccurate since one may easily isolate an $E$. coli population that is up to $80 \%$ persister cells by pre-treating exponentially growing cells with rifampicin, chloramphenicol, or carbonyl cyanide $m$-chlorophenylhydrazone (Kwan et al., 2013). Furthermore, by treating exponentially growing cells via acid or hydrogen peroxide, nearly $100 \%$ of the cells will be persistent (Hong et al., 2012). To argue that these persister cells created by pretreatment are somehow not "bona fide" (Henry and Brynildsen, 2016) and different from persister cells formed after stress, such as the nutrient stress as a result of growth, is disingenuous. Also, heterologous production of any potent toxin or toxic protein makes persister cells at high percentages (Hong et al., 2012; Chowdhury et al., 2016a). More simply, allowing E. coli to reach the stationary phase creates a cell population where almost all the cells are tolerant to $\beta$ lactams (Luidalepp et al., 2011).

\section{Misperception 10: There Are Difficulties in Obtaining the Metabolome or Transcriptome of Persister Cells}

It has also argued in 2016 that there are "difficulties associated with segregating persisters from other cell types have prevented a bona fide transcriptome or metabolome of native persisters to be obtained" (Henry and Brynildsen, 2016) and "Without methods to provide high purity samples, high-throughput (HT) techniques (e.g., RNA sequencing) cannot be employed to provide knowledge of naturally occurring persisters" (Henry and Brynildsen, 2016). These recent claims are inappropriate given that not only has an uniform population of persister cells been created by generating a more toxic toxin of a TA system, but then transcriptomics was used to show that cells that were less fit are more likely to be persisters (Hong et al., 2012). Furthermore, given that persister cells are metabolically inactive, one would expect that the transcriptome of persister cells would not be particularly informative since little (if any) transcription should occur.

\section{Misperception 11: Interspecies and Interkingdom Signal Indole Increases Persistence}

The claim by Vega et al. (2012) that indole increases persistence is erroneous (Hu et al., 2015; Kwan et al., 2015b). Indole is an important signal since it is produced by at least 27 different genera that produce tryptophanase (TnaA) which converts tryptophan into indole (Lee et al., 2007b). Furthermore, indole is one of two quorum-sensing signals (Lee et al., 2007a) in E. coli: indole is the primary signal at low temperatures and autoinducer- 2 is the primary signal at higher temperatures in the gastrointestinal tract (Lee et al., 2008). Indole is also an interspecies signal that decreases pathogenicity (Lee et al., 2009; Chu et al., 2012; Hidalgo-Romano et al., 2014), alters biofilm formation of neighboring cells (Lee et al., 2007b); tightens epithelial cell junctions to reduce infection (Bansal et al., 2010; Shimada et al., 2013), and increases antibiotic resistance by activating efflux pumps (Hirakawa et al., 2005; Kobayashi et al., 2006).

By investigating two different systems, the YafQ/DinJ TA system and cyclic diguanylate (c-di-GMP) signaling, it has been determined that indole instead reduces persistence. The E. coli dinJ-yafQ TA locus is induced in persister cells (Keren et al., 2004), and toxin YafQ increases persister cells with multiple antibiotics including ampicillin (1000-fold) and ciprofloxacin (43-fold; Hu et al., 2015). The mechanism for this increase in persistence is the reduction of TnaA levels since RNase YafQ cleaves TnaA mRNA (which has 16 putative YafQ cleavage sites; $\mathrm{Hu}$ et al., 2015). Adding indole exogenously also reduces persistence up to 52-fold (Hu et al., 2015).

The second set of results showing indole reduces persistence was obtained by studying ci-di-GMP intercellular signaling and finding production of DosP (direct oxygen sensing phosphodiesterase) increases persistence by over a thousand fold (Kwan et al., 2015b). The mechanism of increasing persistence by DosP is through its degradation of another cyclic nucleotide signal, cyclic adenosine monophosphate (cAMP), which results in lower indole levels due to reduced TnaA activity (Kwan et al., 2015b).

Furthermore, substituted indoles reduce persistence (Lee et al., 2016). By testing 36 diverse indole derivatives for the ability to inhibit persister cells of Gram-negative E. coli and Grampositive $S$. aureus, 5-iodoindole, 4-fluoroindole, 7-chloroindole, and 7-bromoindole were identified that eradicate persister cell formation by both bacteria. 5-Iodoindole also diminished the virulence of $S$. aureus. Hence, it is more likely that indole reduces persistence.

\section{Misperception 12: There Are No Persister Mechanisms}

It has been argued recently in 2016 that "the mechanisms that allow one non-growing, stationary-phase cell to become a persister while approximately 90-99 of its genetically identical kin remain sensitive to antibiotic have yet to be determined" (Henry and Brynildsen, 2016). This statement is false, since, as outlined above, transcriptomics have been used to determine 
that cells that are less fit to deal with antibiotic stress more readily become persister cells (Hong et al., 2012), since the mechanisms by which indole decreases persistence have been determined (Hu et al., 2015; Kwan et al., 2015b), and since the relationship between the toxin HipA, ppGpp, and persisters has been established (Korch et al., 2003).

\section{CONCLUSION}

On the surface, it would seem that the study of persister cells should be straight-forward extension of studies on antimicrobials; the reality has been far different in that insights into the mechanisms by which persister cells arise (e.g., indole, TA systems and ppGpp, higher persistence in less fit cells) has been difficult since no one protein is responsible (Chowdhury et al., 2016a; Kaldalu et al., 2016). For example, many studies using transposon-sequencing (Shan et al., 2015), protein expression (Spoering et al., 2006), and gene knockouts ( $\mathrm{Hu}$ and Coates, 2005; Hansen et al., 2008) have failed to produce a coherent mechanism for persister cell formation. One complicating factor is that relatively large changes in persister cell concentrations must occur for a link to be made conclusively to a phenotype; i.e., changes of fivefold or less are probably meaningless when cell populations change by orders of magnitude (e.g., from $10^{9}$ to $10^{4}$ cells $/ \mathrm{mL}$ ). Also, errors in technique abound with many authors waking the persister cells with fresh medium; this leads to the phenotypes of active cells being attributed to persister cells. Another complicating factor is that the

\section{REFERENCES}

Allison, K. R., Brynildsen, M. P., and Collins, J. J. (2011). Metabolite-enabled eradication of bacterial persisters by aminoglycosides. Nature 473, 216-220. doi: 10.1038/nature10069

Amato, S. M., Orman, M. A., and Brynildsen, M. P. (2013). Metabolic control of persister formation in Escherichia coli. Mol. Cell 50, 475-487. doi: 10.1016/j. molcel.2013.04.002

Balaban, N. Q., Merrin, J., Chait, R., Kowalik, L., and Leibler, S. (2004). Bacterial persistence as a phenotypic switch. Science 305, 1622-1625. doi: 10.1126/ science. 1099390

Bansal, T., Alaniz, R. C., Wood, T. K., and Jayaraman, A. (2010). The bacterial signal indole promotes epithelial cell barrier properties and attenuates inflammation. Proc. Natl. Acad. Sci. U.S.A. 107, 228-233. doi: 10.1073/pnas. 0906112107

Bigger, J. W. (1944). Treatment of staphylococcal infections with penicillin by intermittent sterilisation. Lancet 244, 497-500. doi: 10.1016/S0140-6736(00) 74210-3

Brauner, A., Fridman, O., Gefen, O., and Balaban, N. Q. (2016). Distinguishing between resistance, tolerance and persistence to antibiotic treatment. Nat. Rev. Microbiol. 14, 320-330. doi: 10.1038/nrmicro.2016.34

Chowdhury, N., Kwan, B. W., and Wood, T. K. (2016a). Persistence increases in the absence of the alarmone guanosine tetraphosphate by reducing cell growth. Sci. Rep. 6, 20519. doi: 10.1038/srep20519

Chowdhury, N., Wood, T. L., Martínez-Vázquez, M., García-Contreras, R., and Wood, T. K. (2016b). DNA-crosslinker cisplatin eradicates bacterial persister cells. Biotechnol. Bioeng. 113, 1984-1992. doi: 10.1002/bit. 25963

Chu, W., Zere, T. R., Weber, M. M., Wood, T. K., Whiteley, M., HidalgoRomano, B., et al. (2012). Indole production promotes Escherichia coli mixed-culture growth with Pseudomonas aeruginosa by inhibiting field has been side-tracked by concepts such as "potentiation," stochastic/spontaneous generation (and the associated plethora of bet-hedging claims), and metabolic activity as well as sidetracked by the use of E. coli strains such as hipA7 and delta 10. Clearly the HipA toxin was important for establishing the link between TA systems and persister cells and for elucidating the role of ppGpp, but far better systems exist now for studying persisters; i.e., ones with active toxins. Given that microbial infections are the leading cause of death worldwide (Rasko and Sperandio, 2010) and that persister cells are indubitably a cause of recurring infections (Fauvart et al., 2011), discerning more details for the mechanism of persister cell formation is an essential area of research, and one which should be approached with fewer misperceptions.

\section{AUTHOR CONTRIBUTIONS}

TW conceived and wrote the manuscript. J-SK helped write the manuscript.

\section{ACKNOWLEDGMENTS}

This work was supported by the Army Research Office (W911NF14-1-0279). TW is the Biotechnology Endowed Professor at the Pennsylvania State University. The authors appreciate the fruitful discussions with Dr. Stephen Knabel of the Pennsylvania State University.

quorum signaling. Appl. Environ. Microbiol. 78, 411-419. doi: 10.1128/aem. 06396-11

Conlon, B. P., Rowe, S. E., Gandt, A. B., Nuxoll, A. S., Donegan, N. P., Zalis, E. A., et al. (2016). Persister formation in Staphylococcus aureus is associated with ATP depletion. Nat. Microbiol. 1:16051. doi: 10.1038/nmicrobiol.2016.51

Cruz-Muñiz, M. Y., López-Jacome, L. E., Hernández-Durán, M., FrancoCendejas, R., Licona-Limón, P., Ramos-Balderas, J. L., et al. (2016). Repurposing the anticancer drug mitomycin $\mathrm{C}$ for the treatment of persistent Acinetobacter baumannii infections. Int. J. Antimicrob doi: 10.1016/j. ijantimicag.2016.08.022 [Epub ahead of print],

Dörr, T., Vulić, M., and Lewis, K. (2010). Ciprofloxacin causes persister formation by inducing the TisB toxin in Escherichia coli. PLoS Biol. 8:e1000317. doi: 10.1371/journal.pbio.1000317

Fauvart, M., De Groote, V. N., and Michiels, J. (2011). Role of persister cells in chronic infections: clinical relevance and perspectives on anti-persister therapies. J. Med. Microbiol. 60, 699-709. doi: 10.1099/jmm.0.030932-0

Germain, E., Roghanian, M., Gerdes, K., and Maisonneuve, E. (2015). Stochastic induction of persister cells by HipA through (p)ppGpp-mediated activation of mRNA endonucleases. Proc. Natl. Acad. Sci. U.S.A. 112, 5171-5176. doi: 10.1073/pnas.1423536112

Guo, Y., Quiroga, C., Chen, Q., McAnulty, M. J., Benedik, M. J., Wood, T. K., et al. (2014). RalR (a DNase) and RalA (a small RNA) form a type I toxinantitoxin system in Escherichia coli. Nucleic Acids Res. 42, 6448-6462. doi: $10.1093 /$ nar/gku279

Hansen, S., Lewis, K., and Vulic, M. (2008). Role of global regulators and nucleotide metabolism in antibiotic tolerance in Escherichia coli. Antimicrob. Agents Chemother. 52, 2718-2726. doi: 10.1128/aac.00144-08

Harrison, J. J., Wade, W. D., Akierman, S., Vacchi-Suzzi, C., Stremick, C. A., Turner, R. J., et al. (2009). The chromosomal toxin gene yafQ is a determinant of multidrug tolerance for Escherichia coli growing in a biofilm. Antimicrob. Agents Chemother. 53, 2253-2258. doi: 10.1128/aac.00043-09 
Henry, T. C., and Brynildsen, M. P. (2016). Development of Persister-FACSeq: a method to massively parallelize quantification of persister physiology and its heterogeneity. Sci. Rep. 6:25100. doi: 10.1038/srep25100

Hidalgo-Romano, B., Gollihar, J., Brown, S. A., Whiteley, M., Valenzuela, E., Kaplan, H. B., et al. (2014). Indole inhibition of $\mathrm{N}$-acylated homoserine lactone-mediated quorum signalling is widespread in Gram-negative bacteria. Microbiology 160, 2464-2473. doi: 10.1099/mic.0.081729-0

Hirakawa, H., Inazumi, Y., Masaki, T., Hirata, T., and Yamaguchi, A. (2005). Indole induces the expression of multidrug exporter genes in Escherichia coli. Mol. Microbiol. 55, 1113-1126. doi: 10.1111/j.1365-2958.2004.0 4449. $\mathrm{x}$

Hobby, G. L., Meyer, K., and Chaffee, E. (1942). Observations on the mechanism of action of penicillin. Exp. Biol. Med. 50, 281-285. doi: 10.3181/00379727-5013773

Hong, S. H., Wang, X., O'Connor, H. F., Benedik, M. J., and Wood, T. K. (2012). Bacterial persistence increases as environmental fitness decreases. Microb. Biotechnol. 5, 509-522. doi: 10.1111/j.1751-7915.2011. 00327.x

Hu, Y., and Coates, A. R. M. (2005). Transposon mutagenesis identifies genes which control antimicrobial drug tolerance in stationary-phase Escherichia coli. FEMS Microbiol. Lett. 243, 117-124. doi: 10.1016/j.femsle.2004. 11.049

Hu, Y., Kwan, B. W., Osbourne, D. O., Benedik, M. J., and Wood, T. K. (2015). Toxin YafQ increases persister cell formation by reducing indole signalling. Environ. Microbiol. 17, 1275-1285. doi: 10.1111/1462-2920. 12567

Kaldalu, N., Hauryliuk, V., and Tenson, T. (2016). Persisters-as elusive as ever. Appl. Microbiol. Biotechnol. 100, 6545-6553. doi: 10.1007/s00253-0167648-8

Keren, I., Shah, D., Spoering, A., Kaldalu, N., and Lewis, K. (2004). Specialized persister cells and the mechanism of multidrug tolerance in Escherichia coli. J. Bacteriol. 186, 8172-8180. doi: 10.1128/JB.186.24.8172-8180. 2004

Kim, Y., and Wood, T. K. (2010). Toxins Hha and CspD and small RNA regulator Hfq are involved in persister cell formation through MqsR in Escherichia coli. Biochem. Biophys. Res. Commun. 391, 209-213. doi: 10.1016/j.bbrc.2009. 11.033

Kobayashi, A., Hirakawa, H., Hirata, T., Nishino, K., and Yamaguchi, A. (2006). Growth phase-dependent expression of drug exporters in Escherichia coli and its contribution to drug tolerance. J. Bacteriol. 188, 5693-5703. doi: 10.1128/JB. 00217-06

Korch, S. B., Henderson, T. A., and Hill, T. M. (2003). Characterization of the hipA7 allele of Escherichia coli and evidence that high persistence is governed by (p)ppGpp synthesis. Mol. Microbiol. 50, 1199-1213. doi: 10.1046/j.1365-2958. 2003.03779.x

Kwan, B. W., Chowdhury, N., and Wood, T. K. (2015a). Combatting bacterial infections by killing persister cells with mitomycin C. Environ. Microbiol. 17, 4406-4414. doi: 10.1111/1462-2920.12873

Kwan, B. W., Osbourne, D. O., Hu, Y., Benedik, M. J., and Wood, T. K. (2015b). Phosphodiesterase DosP increases persistence by reducing cAMP which reduces the signal indole. Biotechnol. Bioeng. 112, 588-600. doi: 10.1002/ bit. 25456

Kwan, B. W., Valenta, J. A., Benedik, M. J., and Wood, T. K. (2013). Arrested protein synthesis increases persister-like cell formation. Antimicrob. Agents Chemother. 57, 1468-1473. doi: 10.1128/AAC.02135-12

Lee, J., Attila, C., Cirillo, S. L., Cirillo, J. D., and Wood, T. K. (2009). Indole and 7hydoxyindole diminish Pseudomonas aeruginosa virulence. Microb. Biotechnol. 2, 75-90. doi: 10.1111/j.1751-7915.2008.00061.x

Lee, J., Bansal, T., Jayaraman, A., Bentley, W. E., and Wood, T. K. (2007a). Enterohemorrhagic Escherichia coli biofilms are inhibited by 7-hydroxyindole and stimulated by isatin. Appl. Environ. Microbiol. 73, 4100-4109. doi: 10.1128/ AEM.00360-07

Lee, J., Jayaraman, A., and Wood, T. K. (2007b). Indole is an inter-species biofilm signal mediated by SdiA. BMC Microbiol. 7:42. doi: 10.1186/14712180-7-42

Lee, J., Zhang, X.-S., Hegde, M., Bentley, W. E., Jayaraman, A., and Wood, T. K. (2008). Indole cell signaling occurs primarily at low temperatures in Escherichia coli. ISME J. 2, 1007-1023. doi: 10.1038/ismej.2008.54
Lee, J.-H., Kim, Y.-G., Gwon, G., Wood, T. K., and Lee, J. (2016). Halogenated indoles eradicate bacterial persister cells and biofilms. AMB Express 6, 123. doi: 10.1186/s13568-016-0297-6

Luidalepp, H., Jõers, A., Kaldalu, N., and Tenson, T. (2011). Age of inoculum strongly influences persister frequency and can mask effects of mutations implicated in altered persistence. J. Bacteriol. 193, 3598-3605. doi: 10.1128/jb. 00085-11

Maisonneuve, E., Castro-Camargo, M., and Gerdes, K. (2013). (p)ppGpp controls bacterial persistence by stochastic induction of toxin-antitoxin activity. Cell 154, 1140-1150. doi: 10.1016/j.cell.2013.07.048

Maisonneuve, E., Shakespeare, L. J., Jørgensen, M. G., and Gerdes, K. (2011). Bacterial persistence by RNA endonucleases. Proc. Natl. Acad. Sci. U.S.A. 108, 13206-13211. doi: 10.1073/pnas.1100186108

Möker, N., Dean, C. R., and Tao, J. (2010). Pseudomonas aeruginosa increases formation of multidrug-tolerant persister cells in response to quorumsensing signaling molecules. J. Bacteriol. 192, 1946-1955. doi: 10.1128/jb. 01231-09

Moyed, H. S., and Bertrand, K. P. (1983). hipA, a newly recognized gene of Escherichia coli K-12 that affects frequency of persistence after inhibition of murein synthesis. J. Bacteriol. 155, 768-775.

Orman, M. A., and Brynildsen, M. P. (2013). Dormancy is not necessary or sufficient for bacterial persistence. Antimicrob. Agents Chemother. 57, 3230-3239. doi: 10.1128/aac.00243-13

Osbourne, D. O., Soo, V. W. C., Konieczny, I., and Wood, T. K. (2014). Polyphosphate, cyclic AMP, guanosine tetraphosphate, and c-di-GMP reduce in vitro Lon activity. Bioengineered 5, 264-268. doi: 10.4161/bioe.29261

Pecota, D. C., and Wood, T. K. (1996). Exclusion of T4 Phage by the hok/sok killer locus from plasmid R1. J. Bacteriol. 178, 2044-2050. doi: 10.1128/jb.178.7.20442050.1996

Pu, Y., Zhao, Z., Li, Y., Zou, J., Ma, Q., Zhao, Y., et al. (2016). Enhanced efflux activity facilitates drug tolerance in dormant bacterial cells. Mol. Cell 62, 284-294. doi: 10.1016/j.molcel.2016.03.035

Ramisetty, B. C. M., Ghosh, D., Roy Chowdhury, M., and Santhosh, R. S. (2016). What is the link between stringent response, endoribonuclease encoding type ii toxin-antitoxin systems and persistence? Front. Microbiol. 7:1882. doi: 10.3389/ fmicb.2016.01882

Rasko, D. A., and Sperandio, V. (2010). Anti-virulence strategies to combat bacteria-mediated disease. Nat. Rev. Drug Discov. 9, 117-128. doi: 10.1038/ $\operatorname{nrd} 3013$

Schumacher, M. A., Balani, P., Min, J., Chinnam, N. B., Hansen, S., Vulic, M., et al. (2015). HipBA-promoter structures reveal the basis of heritable multidrug tolerance. Nature 524, 59-64. doi: 10.1038/nature14662

Shah, D., Zhang, Z., Khodursky, A., Kaldalu, N., Kurg, K., and Lewis, K. (2006). Persisters: a distinct physiological state of E. coli. BMC Microbiol. 6:53. doi: 10.1186/1471-2180-6-53

Shan, Y., Lazinski, D., Rowe, S., Camilli, A., and Lewis, K. (2015). Genetic basis of persister tolerance to aminoglycosides in Escherichia coli. mBio 6, e78-e15. doi: 10.1128/mBio.00078-15

Shimada, Y., Kinoshita, M., Harada, K., Mizutani, M., Masahata, K., Kayama, H., et al. (2013). Commensal bacteria-dependent indole production enhances epithelial barrier function in the colon. PLoS ONE 8:e80604. doi: 10.1371/ journal.pone.0080604

Spoering, A. L., Vulić, M., and Lewis, K. (2006). GlpD and PlsB participate in persister cell formation in Escherichia coli. J. Bacteriol. 188, 5136-5144. doi: 10.1128/jb.00369-06

Tan, Q., Awano, N., and Inouye, M. (2011). YeeV is an Escherichia coli toxin that inhibits cell division by targeting the cytoskeleton proteins, FtsZ and MreB. Mol. Microbiol. 79, 109-118. doi: 10.1111/j.1365-2958.2010. 07433.x

Van den Bergh, B., Michiels, J. E., Wenseleers, T., Windels, E. M., Boer, P. V., Kestemont, D., et al. (2016). Frequency of antibiotic application drives rapid evolutionary adaptation of Escherichia coli persistence. Nat. Microbiol. 1:16020. doi: 10.1038/nmicrobiol.2016.20

Vega, N. M., Allison, K. R., Khalil, A. S., and Collins, J. J. (2012). Signalingmediated bacterial persister formation. Nat. Chem. Biol. 8, 431-433. doi: 10. 1038/nchembio. 915

Verstraeten, N., Knapen, W. J., Kint, C. I., Liebens, V., Van den Bergh, B., Dewachter, L., et al. (2015). Obg and membrane depolarization are part of a 
microbial bet-hedging strategy that leads to antibiotic tolerance. Mol. Cell 59, 9-21. doi: 10.1016/j.molcel.2015.05.011

Wakamoto, Y., Dhar, N., Chait, R., Schneider, K., Signorino-Gelo, F., Leibler, S., et al. (2013). Dynamic persistence of antibiotic-stressed mycobacteria. Science 339, 91-95. doi: 10.1126/science. 1229858

Wang, X., Lord, D. M., Cheng, H.-Y., Osbourne, D. O., Hong, S. H., SanchezTorres, V., et al. (2012). A Novel type V TA system where mRNA for toxin GhoT is cleaved by antitoxin GhoS. Nat. Chem. Biol. 8, 855-861. doi: 10.1038/ nchembio. 1062

Wood, T. K. (2016). Combatting bacterial persister cells. Biotechnol. Bioeng. 113, 476-483. doi: 10.1002/bit.25721

Wood, T. K., Knabel, S. J., and Kwan, B. W. (2013). Bacterial persister cell formation and dormancy. Appl. Environ. Microbiol. 79, 7116-7121. doi: 10. 1128/AEM.02636-13
Yamaguchi, Y., Park, J., and Inouye, M. (2011). Toxin-antitoxin systems in bacteria and archaea. Annu. Rev. Genet. 45, 61-79. doi: 10.1146/annurev-genet-110410132412

Conflict of Interest Statement: The authors declare that the research was conducted in the absence of any commercial or financial relationships that could be construed as a potential conflict of interest.

Copyright $\odot 2016$ Kim and Wood. This is an open-access article distributed under the terms of the Creative Commons Attribution License (CC BY). The use, distribution or reproduction in other forums is permitted, provided the original author(s) or licensor are credited and that the original publication in this journal is cited, in accordance with accepted academic practice. No use, distribution or reproduction is permitted which does not comply with these terms. 